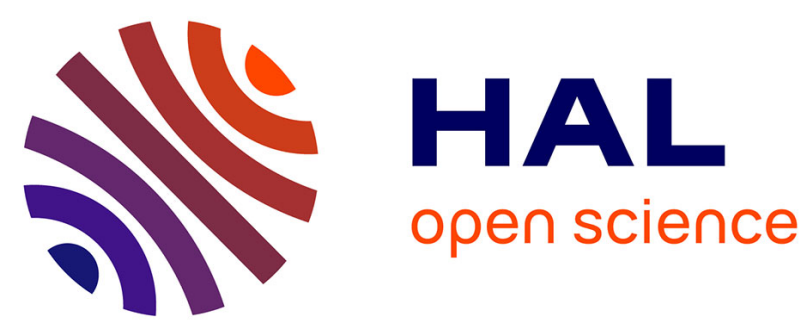

\title{
A Correlation Retina for Real-Time Pattern Recognition
}

Bernard Lamalle, Guy Cathébras, L.F.C. Lew Yan Voon, Patrick Gorria, Benaïssa Bellach, Olivier Aubreton

\section{To cite this version:}

Bernard Lamalle, Guy Cathébras, L.F.C. Lew Yan Voon, Patrick Gorria, Benaïssa Bellach, et al.. A Correlation Retina for Real-Time Pattern Recognition. ETFA: Emerging Technologies and Factory Automation, Oct 2001, Antibes-Juan les Pins, France. pp.367-372, 10.1109/ETFA.2001.997708 . lirmm-02306537

\section{HAL Id: lirmm-02306537 https://hal-lirmm.ccsd.cnrs.fr/lirmm-02306537}

Submitted on 6 Oct 2019

HAL is a multi-disciplinary open access archive for the deposit and dissemination of scientific research documents, whether they are published or not. The documents may come from teaching and research institutions in France or abroad, or from public or private research centers.
L'archive ouverte pluridisciplinaire $\mathbf{H A L}$, est destinée au dépôt et à la diffusion de documents scientifiques de niveau recherche, publiés ou non, émanant des établissements d'enseignement et de recherche français ou étrangers, des laboratoires publics ou privés. 


\title{
A Correlation Retina for Real-Time Pattern Recognition
}

\author{
B. Lamalle ${ }^{\dagger}$, G. Cathebras ${ }^{\ddagger}$, L.F.C. Lew Yan Voon ${ }^{\dagger}$, P. Gorria ${ }^{\dagger}$, B. Bellach ${ }^{\dagger}$ and O. Aubreton ${ }^{\dagger}$ \\ †Laboratoire LE2I - Université de Bourgogne \\ I.U.T. Le Creusot, 12, rue de la fonderie, 71200 Le Creusot \\ FRANCE \\ ${ }^{\ddagger}$ LIRMM - Université Montpellier II \\ 161, rue Ada, 34392 Montpellier Cedex 5 \\ FRANCE
}

\begin{abstract}
We present in this paper a programmable real-time pattern recognition retina fabricated in standard CMOS $0.6 \mu \mathrm{m}$ process. Each of the pixels of the retina is composed of a photodiode and an electronic device used during the programming phase to digitalize the image of the pattern to recognize into a binary image stored in latches. The array of pixels is thus partitioned into two complementary disjoint subsets with all the photodiodes of the same sub-set connected together in order to obtain the sum total of the currents. During the analysis phase, an optical correlation between the projected image and the reference binary image memorized in the circuit is done. The result is read-out as two voltages representing the following two currents: a "white" current proportional to the luminous flux falling on the photodiodes pertaining to the "white" part of the binary reference image and a "black" current corresponding to the black part. By comparing these two voltages to expected values, a shift of the pattern or a difference between the observed and programmed pattern can be detected.
\end{abstract}

\section{INTRODUCTION}

A silicon retina is a particular image sensor in which an analog and/or digital signal processing circuitry is integrated next to the photosensitive or image sensing element. The role of the latter is to allow some low level processing to be done on the image signals before they are made available at the output of the circuit $[1,2,3,4,5]$. The type of processing that we would like to implement in the case of our retina is pattern recognition in real time. Our idea is to compare an observed image with a reference binary image programmed in the retina. The result of the comparison is two signals in the form of electrical currents that would allow the retina to detect a shift or a difference between the observed image and the programmed reference image.

In the next section of the paper we shall present the working principle of the retina and show its pattern recognition features by use of a simple example. In section III, we shall describe the architecture of the circuit and give the block diagrams of the programmable pixel from which an array of $100 \times 100$ pixels has been fabricated in standard CMOS $0.6 \mu \mathrm{m}$ process. In section IV, we shall describe the characterization of the retina and give the results that we have obtained. Finally, the results of the experiments that we have carried out on the retina in order to verify its ability to recognize patterns or to detect spatial shifts of patterns will be reported in section $\mathrm{V}$ before concluding by section VI.

\section{WORKING PRINCIPLE OF THE RETINA}

The working principle of our silicon retina is based on the comparison between an image projected on the circuit by some optical means and a reference binary image obtained by thresholding the image to recognize according to a suitable threshold as shown in Figure 1. This reference binary image is stored in latches in the circuit by assigning the logic value 0 to a black pixel and the logic value 1 to a white pixel $[6,7,8]$.

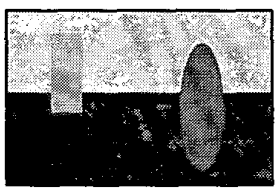

a) Image to recognize

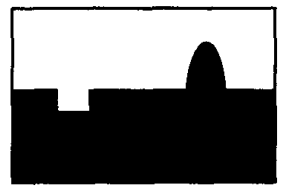

b) Binary reference image $R(i, j)$
Figure 1: Binary reference image

When an image is projected on the retina, the currents produced by all the pixels pertaining to respectively the black and white areas of the reference binary image are summed in order to give two total currents called as the black and white pixel currents. These currents, denoted by $I_{\text {black }}$ and $I_{\text {white, }}$ can be expressed as:

$$
I_{\text {white }}=K \sum_{i=0}^{N-1} \sum_{j=0}^{N-1} R(i, j) \cdot X(i, j)
$$

and

$$
I_{\text {black }}=K \sum_{i=0}^{N-1} \sum_{j=0}^{N-1} \overline{R(i, j)} \cdot X(i, j),
$$

where $X(i, j)$ is the luminous flux falling on the pixel of coordinates $(i, j)$ and $K$ a constant which defines the linear relationship between the luminous flux and the current produced by the pixel. 
Now, let us consider the two images represented in Figure 2.a) and Figure 3.a) as well as the $I_{\text {black }}$ and $I_{\text {white }}$ currents computed for these two images using a binary reference image obtained by thresholding the first image (e.g. the image of Figure 2.a)). One would notice here that this image is the same as that of Figure 1.a) so that the binary reference image is the one represented in Figure 1.b).

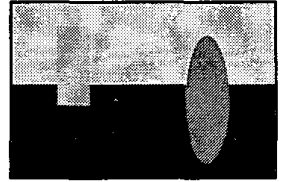

a) Observed image: $X(i, j)$

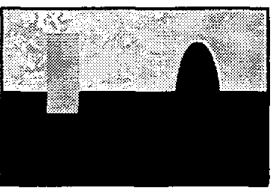

b) $I_{\text {white }}$

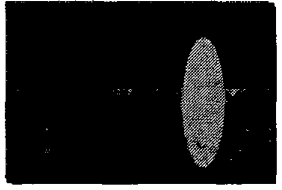

c) $I_{\text {black }}$
Figure 2: Computing the $I_{\text {white }}$ and $I_{\text {black }}$ currents for the reference image

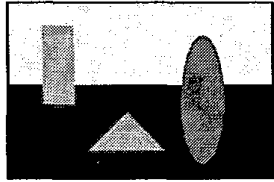

a) Observed image: $X^{\prime}(i, j)$

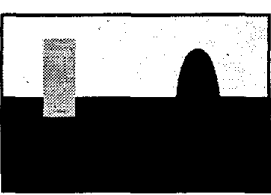

b) $I_{\text {white }}=I_{\text {white }}$

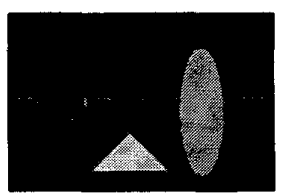

c) $\mathrm{I}_{\text {black }}>\mathrm{I}_{\text {black }}$
Figure 3: Computing the $I_{\text {white }}$ and $I_{\text {black }}$ currents for the image to compare

It is obvious from Figure 2 and Figure 3 that the $I_{\text {white }}$ current is the same for both images whereas the $I_{\text {black }}$ current is different due to the presence of the triangle. Thus, by comparing the $I_{\text {black }}$ and $I_{\text {white }}$ currents, we can conclude that the images of Figure 2.a) and Figure 3.a) are different. In this particular case, only the $I_{\text {black }}$ current is involved in the discrimination of the two images as illustrated in Figure 4 . In the general case, both the $I_{\text {black }}$ and $I_{\text {white }}$ currents contribute to the discrimination of the images.

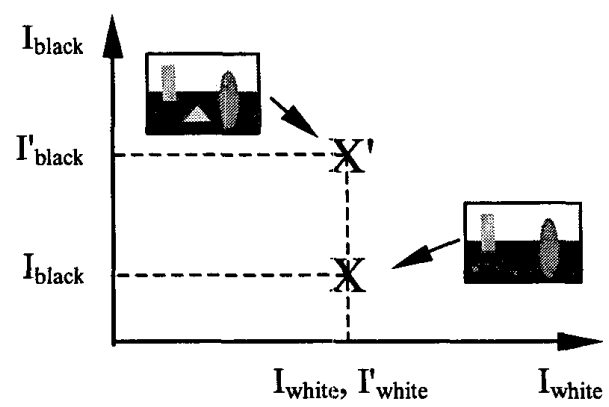

Figure 4: The $I_{\text {black }} / I_{\text {white }}$ plane

\section{THE PROGRAMMABLE RETINA}

The programmable retina is composed of an array of programmable pixels. Each of the pixels contains a photodiode and an electronic device used to program the reference image by digitalizing it and storing the binary value in a latch. According to this value the photodiode is connected to either the $I_{\text {white }}$ or the $I_{\text {black }}$ current output of the array. The block diagram of the programmable pixels is shown in Figure 5.

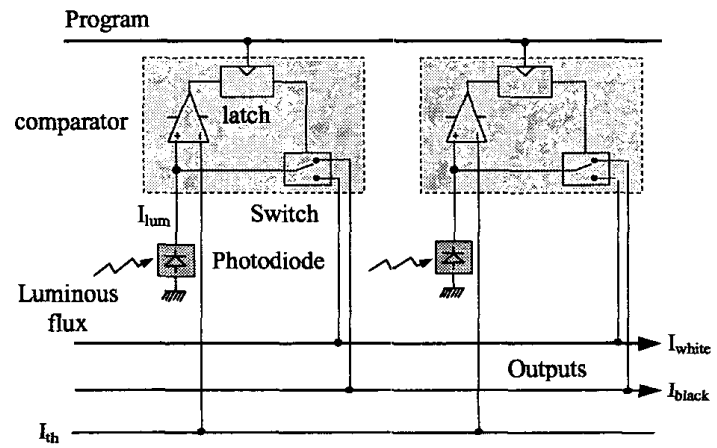

Figure 5: Block diagram of a programmable pixel

During the programming phase, the image to recognize is projected on the circuit and the current produced by each of the photodiodes due to the luminous flux is compared to a threshold current $I_{t h}$ using a current comparator. The result of the comparison is a binary value stored in a latch by applying a pulse to the input "Program" (Figure 5). This binary value is used during normal functioning mode to set the position of the switch in order to connect together all the cathodes pertaining to the same type of photodiodes. The state of the switch is maintained as long as no new memorization or programming pulse is applied.

The pixel has been realized in standard CMOS $0.6 \mu \mathrm{m}$ process with three layers of metal. The size of the pixel is about $50 \times 50 \mu \mathrm{m}$ with a fill factor greater than $30 \%$. From this pixel a correlation retina of $100 \times 100$ pixels has been fabricated. The size of the retina is about $6 \times 6 \mathrm{~mm}$. 


\section{CHARACTERIZATION}

The $I_{\text {black }}$ and $I_{\text {white }}$ currents obtained at the output of the array of pixels are converted into voltages, that we shall denote by $V_{\text {black }}$ and $V_{\text {white, }}$, in order to facilitate their measurement. The current-to-voltage converters, made up of an operational amplifier and a $2.2 \mathrm{M} \Omega$ feedback resistor, are integrated into the retina circuit.

\section{A Spectral response}

A tunable monochromatic light source capable of emitting light rays of wavelength in the range of 300 to $1200 \mathrm{~nm}$ has been used. The determination of the spectral response, represented in Figure 6, is done in two steps:

- Measurement of the spectral characteristic of the light source. A bolometer is used to measure the radiant flux emitted by the monochromatic light source.

- Measurement of the characteristic of the retina. For each wavelength, two voltmeters are used to measure the $\mathrm{V}_{\text {black }}$ and $\mathrm{V}_{\text {white }}$ voltages.

The sensitivity of the sensor, defined as the ratio of the output currents over the radiant flux, can be deduced from these two series of measurements. Note that our measurements give the output voltages, $V_{\text {black }}$ and $V_{\text {white, }}$ instead of the output currents, $I_{\text {black }}$ and $I_{\text {white. }}$. However, the latter can be deduced from the $V_{\text {black }}$ and $V_{\text {white }}$ voltages knowing the trans-resistance value of the current-tovoltage converter.

The sensitivity is a function of the wavelength and for a wavelength of $550 \mathrm{~nm}$, for example, it has been found to be equal to $0.23 \mathrm{~A} / \mathrm{W}$, which corresponds to an efficiency of $51 \%$. This value is to be compared to the $0.2 \mathrm{~A} / \mathrm{W}$ value given in the literature and which corresponds to an efficiency of $41 \%$.

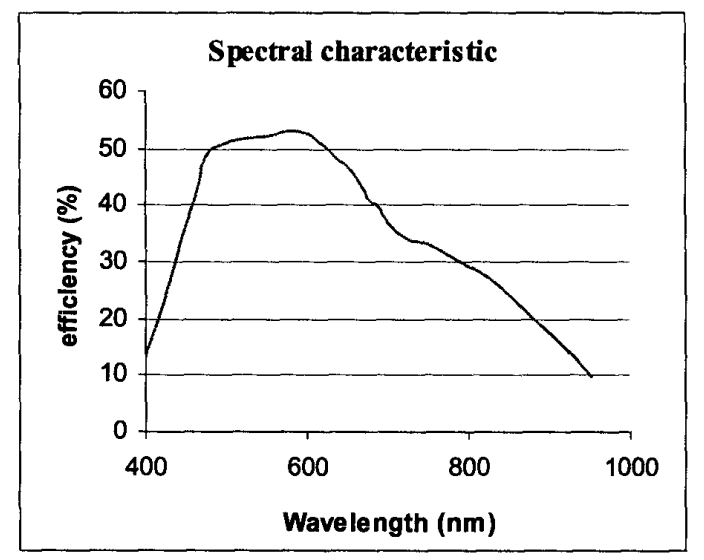

Figure 6: Spectral response of the retina

\section{B Spatial sensitivity}

We are interested here in the minimal surface area, $\Delta S$, of an object that the retina can detect. To determine this surface area, we have placed the retina in the dark and measured the output voltage. It has been found to be equal to $20 \mathrm{mV}$. This value corresponds to the smallest signal, $\mathrm{V}_{\min }$, that can be measured and it is due to the dark current and noise of the device. For a radiant flux of $16 \mu \mathrm{W}$ (the radiant flux of the light source we shall be using for our experiments in the next section) and for $\mathrm{V}_{\min }=20 \mathrm{mV}$, we deduce that the minimal surface area is 60 pixels.

\section{Read-out rate}

The working principle of the array of pixels is based on an optical correlation. The settling time of the $I_{\text {black }}$ and $I_{\text {white }}$ currents is thus only limited by the sum of the generation time of the charge carriers due to the luminous flux falling on the photosensitive area of the pixels and the transit time for these charge carriers to travel from the photosite to the output of the array of pixels. However, the current-to-voltage conversion stage limits the response time of the retina.

To illustrate this, we have memorized an image corresponding to a fan with the three blades in a given position. Next, we have switched on the fan and observed the variation of the read-out $V_{\text {black }}$ and $V_{\text {white }}$ voltages with respect to the rotation speed of the blades. The cutoff frequency of the output stage has been measured and found to be equal to $720 \mathrm{~Hz}$. This cutoff frequency depends on the characteristics of the trans-resistance amplifier or current-to-voltage converter. It can easily be improved if necessary.

\section{$D$ Influence of Fixed Pattern Noise}

Fixed Pattern Noise (FPN) is defined as the noise signals due to a shift in the characteristics of the pixels. Indeed, if the characteristic of any two pixels is slightly different, then the current produced by these two pixels, illuminated by the same luminous flux, will also be slightly different. In order to show the effect of the FPN on the binary image stored in the retina, we have memorized the nine gray levels pattern represented in Figure 7 with the threshold current set to the average current value of the image.

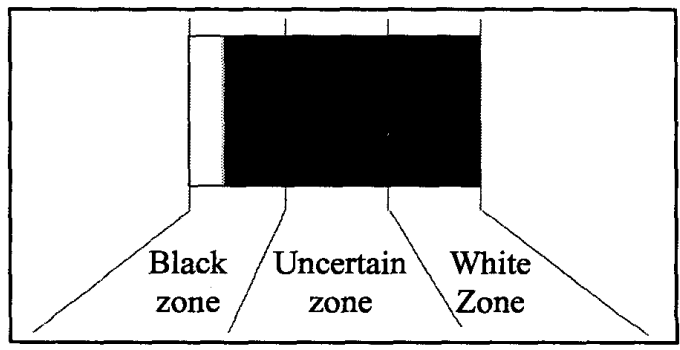

Figure 7: Uncertain zone due to Fixed Pattern Noise 
Without FPN the pixels would be classified into a black and a white zone separated by a well-defined vertical frontier. However, due to FPN we notice that for gray levels for which the corresponding pixel current is close in value to the threshold current, the pixel is randomly classified as a black or white pixel. In the case of the nine gray levels pattern, which we have used for our experiment, we have determined the uncertain zone represented in Figure 7.

\section{APPLICATION}

The experimental set up shown in Figure 8 is used to test the pattern recognition and spatial shift detection features of the retina [9]. The latter is fixed in a case with a $25 \mathrm{~mm}$ lens and located at a distance of $45 \mathrm{~cm}$ from the light source (a $150 \mathrm{~W}$ backlight emitting a maximal radiant flux of $16 \mu \mathrm{W})$. With this set up the view area is $16 \times 16 \mathrm{~cm}$.

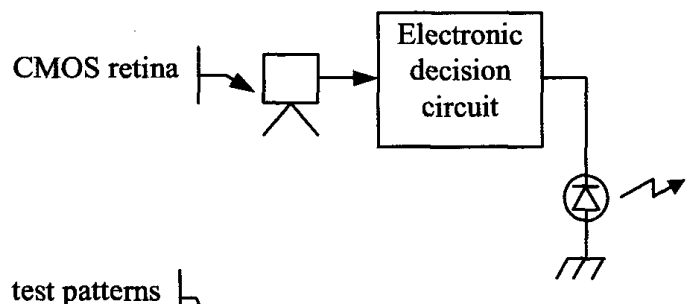

test patterns

Backlight

Figure 8: Experimental set up for testing the retina

Binary test patterns of simple geometrical shapes realized on transparencies have been considered. Any of the test patterns can be used as the reference image that is first programmed in the circuit. Then, the same or a different test pattern is projected onto the retina for comparison, and the two output voltages $\mathrm{V}_{\text {black }}$ and $\mathrm{V}_{\text {white }}$ are processed by an electronic circuit in order to give a binary decision.

When the reference object or image is analyzed after the system has been programmed with this image, we obtain, in the $V_{\text {black }} / V_{\text {white }}$ plane, a point of coordinates $\left(V_{b 0}, V_{w 0}\right)$ that is characteristic of the reference image (see Figure 9.a))

Now, if a test image different from the reference image is analyzed it will be characterized by a point of coordinates different from $\left(\mathrm{V}_{\mathrm{b} 0}, \mathrm{~V}_{\mathrm{w} 0}\right)$. The spatial distance between the two points is a measure of the difference or spatial shift between the two images.

In order to determine whether two images are the same, different or same and spatially shifted, we have defined the following metric:
$\varepsilon=\left|V_{\text {white }}-V_{\text {w0 }}\right|+\left|V_{\text {black }}-V_{b 0}\right|$

The two images are considered to be the same image and perfectly aligned if $\varepsilon<V_{\text {thl }}$, a predefined threshold voltage. In this case, the characteristic point of the image under analysis $\left(V_{\text {black, }}, V_{\text {white }}\right)$ will be found within the shaded area of the $V_{\text {black }} / V_{\text {white }}$ plane or the shaded area of the $\left|\Delta \mathrm{V}_{\text {black }}\right| /\left|\Delta \mathrm{V}_{\text {white }}\right|$ plane if the absolute difference value is considered (see Figure 9.b)).

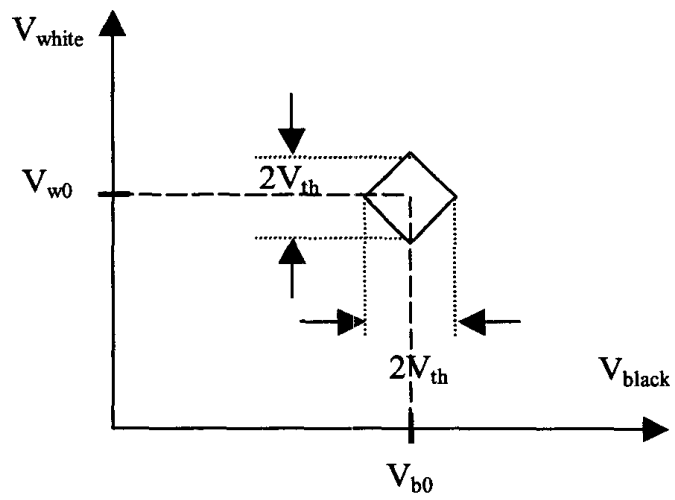

a)

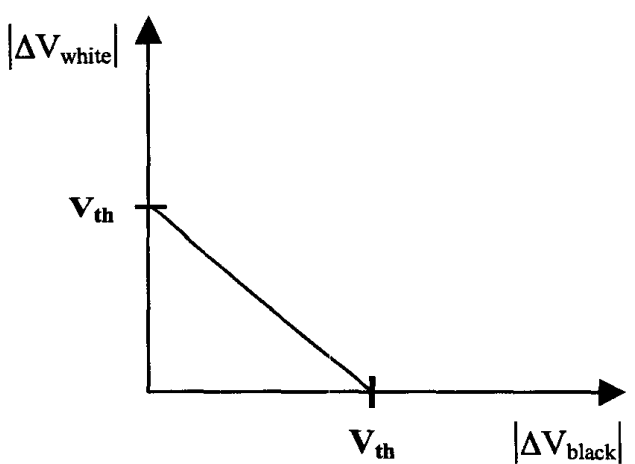

b)

Figure 9: a) $\mathrm{V}_{\text {white }} / \mathrm{V}_{\text {bhack }}$ plane; b) $\left|\Delta \mathrm{V}_{\text {white }} /\right| \Delta \mathrm{V}_{\text {black }} \mid$ plane

The block diagram of the electronic decision circuit is represented in Figure 10. In this circuit, a Sample and Hold (S\&H) circuit is used to memorize the reference voltages $\mathrm{V}_{\mathrm{b} 0}$ and $\mathrm{V}_{\mathrm{w} 0}$ corresponding to the reference image. During the analysis phase the error voltage $\varepsilon$ is determined and then compared to the threshold value $V_{\text {th }}$ in order to produce a binary decision result. 


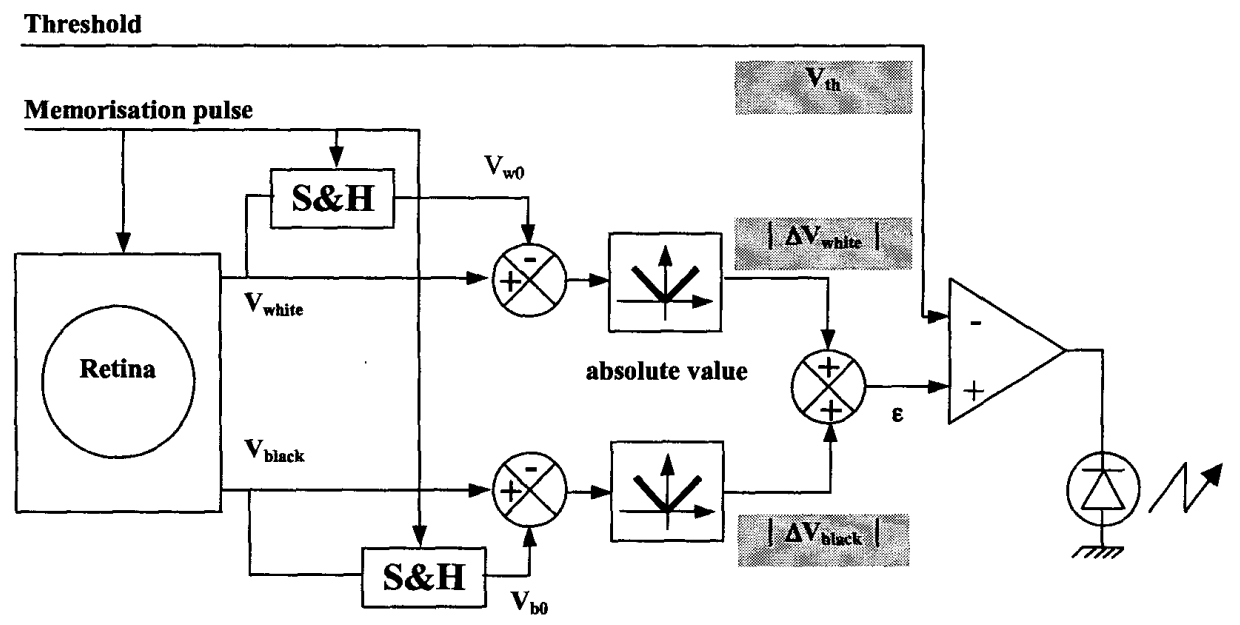

Figure 10: Electronic decision circuit

We have applied to our pattern recognition or spatial shift detection system a set of binary test images composed of simple black geometric shapes over a white background such as squares, circles, triangles, etc. The system has been found to be able to distinguish the different shapes.

In order to quantify the minimum detectable spatial shift, we have memorized a heaviside function which is next shifted in a direction perpendicular to the black to white transition line. We have found that the smallest detectable shift is about $1 \mathrm{~mm}$. According to the characteristics of our experimental set up, in particular the view area, this image shift of $1 \mathrm{~mm}$ corresponds to a pixel shift on the retina of 1 pixel in the direction of the shift. The number of pixels involved in the shift is thus 100 since the array is composed of $100 \times 100$ pixels. This result is consistent with the minimum detectable surface area of at least 60 pixels found in the previous section.

For an in-depth characterization of our retina, we are currently conducting more experiments with gray level patterns of complex shapes. Moreover, we are also studying the application of our device to the position control of an object using two retinas. The idea is the simultaneous control of the horizontal and vertical positions of the object with respect to a reference point.

\section{CONCLUSION}

We have presented in this paper a programmable retina with real-time pattern recognition and spatial shift detection features. The working principle of the circuit is based on the classification (or programming) of the pixels into either black or white pixels representing a binary reference image, and on the determination of the total current produced by all the pixels of the same type when an image is projected on the circuit.
The programming of the pixels into either black or white pixels is achieved by first projecting the reference image on the retina. Then, the current produced by each of the pixels is compared to a threshold value. If this current is greater than the threshold value then the pixel is considered as a white pixel, otherwise it is considered as a black pixel.

During the analysis phase, an optical correlation between the observed image and the memorized binary reference image is realized and the result read-out in the form of two voltages obtained by converting the sum of the currents due to the black and white pixels. By comparing these voltages to reference values, we can decide whether the projected image is similar or not to the reference image used to program the pixels.

A $100 \times 100$ pixels retina based on this working principle has been fabricated in standard CMOS $0.6 \mu \mathrm{m}$ process. Its size is about $6 \times 6 \mathrm{~mm}$ with a fill factor of more than $30 \%$.

The retina circuit has been characterized with respect to its spectral response, spatial sensitivity, read-out rate and influence of Fixed Pattern Noise on the binary image stored in the latches. The spectral response is in agreement with the one found in the literature. Concerning the spatial sensitivity and the read-out rate they are dependent on the experimental conditions. In our case, we have found that the minimal detectable surface area is 60 pixels and the cutoff frequency is $720 \mathrm{~Hz}$.

Simple preliminary experiments done on the retina circuit using binary images have shown that the retina is capable of discriminating simple geometrical shapes such as a square, a circle or a triangle, or detecting a spatial shift of these shapes. The ability of our retina to discriminate between the observed image and the memorized image or to detect spatial shifts depends also on the experimental 
conditions as well as the Fixed Pattern Noise. For our experiments, we have found, using a heaviside function, that any spatial shift that involves more than 100 pixels would be detected. This result is in agreement with the minimal detectable surface area of 60 pixels determined during the characterization of the retina.

\section{REFERENCES}

1. A. Moini, "Vision Chips", Kluwer Academic Publishers, 2000, ISBN: 0-7923-8664-7.

2. Y. Ni, F. Devos, M. Boujrad and J.H. Guan, "Histogram-Equalization-Based Adaptive Image Sensor for Real Time Vision", IEEE Journal of Solid State Circuits, Vol. 32, No. 7, July 1997, pp. 10271036.

3. C.F. Chiu, C.Y. Wu, "The Design of RotationInvariant Pattern Recognition Using the Silicon Retina", IEEE Journal of Solid-State Circuits, Vol. 32, No. 4, April 1997, pp. 526-534.

4. S. Kawahito, M. Yoshida, M. Sasaki, K. Umehara, D. Miyazaki, Y. Tadokoro, K. Murata, S. Doushou and A. Matsuzawa, "A CMOS Image Sensor with Analog Two-Dimensional DCT-Based Compression Circuits for One-Chip Cameras", IEEE Journal of Solid State Circuits, Vol. 32, No. 12, December 1997, pp. 20302041.

5. A. Moini, A. Bouzerdoum, K. Eshraghian, A. Yakovleff, X.T. Nguyen, A. Blanksby, R. Beare, D. Abbott and R.E. Bogner, "An Insect Vision-Based Motion Detection Chip", IEEE Journal of Solid-State Circuits, Vol. 32, No. 2, February 1997, pp. 279-284.

6. P. Gorria, B. Lamalle and G. Cathebras, "Dispositif d'intercorrélation d'une image", Patent $N^{\circ} 9900008$, January 04, 1999.

7. B. Lamalle, P. Gorria, L.F.C. Lew Yan Voon and G. Cathebras, "The Study and Design of a Programmable Silicon Retina for Real Time Pattern Recognition", Proceedings of EOS/SPIE Symposium on Applied Photonics, Thistle Hotel, Glasgow, SCOTLAND, UK, 22-25 May 2000, Vol. 4076, pp 225-234.

8. L.F.C. Lew Yan Voon, B. Lamalle, P. Gorria, G. Cathebras, B. Bellach and D. Navarro, "RealTime Pattern Recognition Retina in CMOS Technology", Proceedings of the 2001 International Conference on Quality Control by Artificial Vision QCAV'2001, Le Creusot, FRANCE, 21-23 May 2001, Vol. 1, pp 238-242.

9. O. Aubreton, "Etude, caractérisation et mise en cuvre d'une rétine d'intercorrélation", Rapport de stage de D.E.A., Laboratoire LE2I, Université de Bourgogne, Juin 2001.

10. S.K. Mendis, S.E. Kemery, R.C. Gee, B. Pain, C.O. Staller Q. Kim and E.R. Fossum, "CMOS Active
Pixel Image Sensors for Highly Integrated Imaging Systems", IEEE Journal of Solid-State Circuits, Vol. 32, No. 2, February 1997, pp. 187-197.

11. O. Yadid-Pecht, B. Pain, C. Staller, C. Clark and E. Fossum, "CMOS Active Pixel Sensor Star Tracker with Regional Electronic Shutter", IEEE Journal of Solid-State Circuits, Vol. 32, No. 2, February 1997, pp. 285-288.

12. Y. lida, E. Oba, K. Mabuchi, N. Nakamura and H. Miura, "A1/4-Inch 330k. Square Pixel Progressive Scan CMOS Active Pixel Image Sensor", IEEE Journal of Solid-State Circuits, Vol. 32, No. 11, November 1997, pp. 2042-2047.

13. R. Wodnicki, G.W. Roberts and M.D. Levine, "A Log-Polar Image Sensor Fabricated in a Standard 1.2- $\mu \mathrm{m}$ ASIC CMOS Process", IEEE Journal of Solid-State Circuits, Vol. 32, No. 8, August 1997, pp. 1274-1277.

14. S. Decker, D. Mac Graph, K. Bremer and C.G. Sodini, "A 256x256 CMOS Array with Wide Dynamic Range Pixels and Column-Parallel Digital Output", IEEE Journal of Solid-state Circuits, Vol. 33, No. 12, December 1997, pp. 2081-2091. 